\title{
4D-DSA: Development and Current Neurovascular Applications
}

\author{
(D) K.L. Ruedinger, (D)S. Schafer, (D) M.A. Speidel, and (D).M. Strother
}

\begin{abstract}
SUMMARY: Originally described by Davis et al in 2013, 4D-Digital Subtraction Angiography (4D-DSA) has developed into a commercially available application of DSA in the angiography suite. 4D-DSA provides the user with 3D time-resolved images, allowing observation of a contrast bolus at any desired viewing angle through the vasculature and at any time point during the acquisition (any view at any time). 4D-DSA mitigates some limitations that are intrinsic to both 2D- and 3D-DSA images. The clinical applications for 4D-DSA include evaluations of AVMs and AVFs, intracranial aneurysms, and atherosclerotic occlusive disease. Recent advances in blood flow quantification using 4D-DSA indicate that these data provide both the velocity and geometric information necessary for the quantification of blood flow. In this review, we will discuss the development, acquisition, reconstruction, and current neurovascular applications of 4D-DSA volumes.
\end{abstract}

ABBREVIATION: TDC $=$ time-density curve

$\mathrm{n}$ many clinical scenarios, multidetector CT angiography and MRA are adequate for endovascular diagnosis and treatmentplanning. However, for complex vascular abnormalities such as aneurysms and AVMs/AVFs, DSA is superior to CTA and MRA due to its improved spatial and temporal resolution. ${ }^{1,2}$ The intracranial vessels most applicable to DSA range in size from 4 to $5 \mathrm{~mm}$ (internal carotid artery) to $<1 \mathrm{~mm}$ (ie, the ophthalmic and anterior choroidal arteries, and the second-division branches and perforators of the middle cerebral artery). These vessels are tortuous and lie in very close proximity to one another; often, they overlap to such a degree that it is challenging to obtain a $2 \mathrm{D}$ projection or a 3D volume free of vascular overlap on the display.

In 1997, Fahrig et $\mathrm{al}^{3}$ showed the feasibility of using the projections from 2 rotational acquisitions (one to acquire a mask, the other to acquire projections containing contrast) to obtain a single $3 \mathrm{D}$ vascular volume (3D-DSA). ${ }^{4}$ Quickly, the clinical utility of 3DDSA became apparent. The ability to view the vasculature from any desired angle and with postprocessing capabilities allowing it to be viewed either as a surface-rendered volume or as multiplanar images, offered obvious advantages over 2D projections. However,

Received July 7, 2020; accepted after revision July 30.

From the School of Medicine and Public Health (K.L.R.) and Departments of Biomedical Engineering (K.L.R.), Medical Physics (M.A.S.), and Radiology (C.M.S.),

University of Wisconsin-Madison, Madison, Wisconsin; and Siemens Healthineers (S.S.), Malvern, Pennsylvania.

Please address correspondence to Katrina Ruedinger, MS, c/o Charles Strother, Department of Radiology, WIMR, 1111 Highland Ave, Madison, WI 53705; e-mail: klruedinger@wisc.edu; @Katrina_Rued

- Indicates open access to non-subscribers at www.ajnr.org

http://dx.doi.org/10.3174/ajnr.A6860 even with these advantages, the lack of temporal information in the $3 \mathrm{D}$ volume still made it impossible to visualize key characteristics of some abnormalities, eg, the interior features of an AVM nidus or the sequence of blood flow entering and leaving an abnormality.

In 2013, Davis et $\mathrm{al}^{5}$ described a reconstruction technique that provided a series of fully time-resolved vascular volumes derived from the projections of a conventional 3D-DSA rotational acquisition. Application of this algorithm generated a time-resolved 3DDSA, also called 4D-DSA. A 4D-DSA volume can not only be viewed at any desired angle but may also be viewed at any desired time during the passage of a contrast bolus through the vasculature. This volume is achieved using the same $\mathrm{x}$-ray exposure and contrast medium dose that is required to create a single 3D-DSA volume, therefore providing more information with the same parameters.

In the following pages, we will describe and illustrate the following: 1) the development and evolution of DSA from 2D to 3D and $3 \mathrm{D}$ to $4 \mathrm{D}, 2)$ the injection and acquisition protocol used to obtain a $4 \mathrm{D}-\mathrm{DSA}, 3$ ) the reconstruction workflow to create a $4 \mathrm{D}$ vascular volume, 4) the current clinical utility of $4 \mathrm{D}-\mathrm{DSA}, 5)$ the use of 4D-DSA for calculation of velocity and flow, and 6) current limitations to and helpful solutions for 4D-DSA.

\section{Development and Evolution of 4D-DSA}

In 1997, Fahrig et $\mathrm{al}^{3}$ used an image intensifier to reconstruct a 3D vascular volume from projections acquired with $2 \mathrm{C}$-arm rotations, leading to broad acceptance of the 3D-DSA technology once flat panel detectors became readily available. ${ }^{4}$ The increased 
size and high spatial resolution of these detectors $(<0.2-\mathrm{mm}$ pixel side length), in combination with intra-arterial contrast injections, allowed volumetric depiction of vascular abnormalities showing anatomic relationships to adjacent structures and visualization of implanted devices. ${ }^{6,7}$ Today, in clinical practice when diagnosing and treating complex cerebrovascular abnormalities, a combination of 2D- and 3D-DSA is used to depict the complex vascular abnormalities (3D) and analyze the dynamic information about the passage of contrast medium from arteries into veins (2D). In most instances though, the topography of these conditions is such that this combination still does not remove the limitations caused by vascular overlap. For example, while both $2 \mathrm{D}$ and $3 \mathrm{D}$ images typically provide excellent information about the major arteries and veins supplying an AVM, neither allow detailed assessment of the angioarchitecture of the nidus of the AVM, eg, the presence of intranidal aneurysms or direct AVFs.

Methods aimed at mitigating this limitation by combining the benefits of both 2D- and 3D-DSA have been proposed since the early 2000s. Schmitt et $\mathrm{al}^{8}$ developed a method encoding bolus arrival time from $2 \mathrm{D}$-DSA images onto a static 3D-DSA, but each acquisition was acquired separately. Copeland et $\mathrm{al}^{9}$ furthered this approach by encoding the contrast passage from biplane 2D-DSA onto a 3D-DSA. Mistretta ${ }^{10}$ and Davis et $\mathrm{al}^{5}$ combined the above approaches, altering the 3D-DSA contrast injection protocol to that of 2D-DSA and started injections at the beginning of a $3 \mathrm{D}$ acquisition. Very quickly, this protocol was modified so that the contrast medium was injected $0.5-1$ second after the start of an acquisition. Encoding this temporal information about the passage of a contrast bolus on the 3D-DSA allowed reconstruction of fully time-resolved 4D-DSA. This method became commercially available in 2015 (Siemens). Although the availability of a time-resolved $3 \mathrm{D}$ volume diminishes some of the problems caused by vascular overlap, it does not completely resolve this issue. During the rotational acquisition of a 4D-DSA, some degradation of temporal information may occur in any of the $2 \mathrm{D}$ projections, ie, when a ray from the source passes through opacified vessels at the same time on its way to the detector.

\section{D-DSA Acquisition Technique and Injection Protocol}

The acquisition technique of a 4D-DSA is generalized into 2 steps: 1) a C-arm rotational acquisition without contrast injection (mask), and 2) a second acquisition with contrast injection (fill). 4D-DSA is designed to allow visualization of the passage of contrast through the $3 \mathrm{D}$ vasculature for inspection. For facilitating this process, a contrast-injection protocol was designed to start at the same time or just after (0.5-1 second) the start of the fill acquisition and to stop before the end of the rotation, thereby visualizing both inflow and outflow of blood/contrast. Consideration should be given to the type of vascular abnormality, eg, AVM or stenosis, as well as the patient's cardiac status, eg, normal or abnormal stroke volume.

Accurate geometric reconstruction of the vasculature for 3DDSA requires that projections be available from about $200^{\circ}$ of rotation. However, to ensure the accuracy of both the geometric and temporal information of a $4 \mathrm{D}$-DSA, it was necessary for the angular range to be increased to at least $260^{\circ}$ so that different parts of the vasculature, ie, arteries and veins, are all opacified for the required $200^{\circ}$. In research and clinical practice, a 6- or 12 -second $260^{\circ}$ fill acquisition during contrast injection is used in accordance with a noninjection mask acquisition. The setup for contrast delivery is the same as for 2D- and 3D-DSA, with appropriate arterial access, catheters, and contrast medium. For a $4 \mathrm{D}$ DSA, the contrast injection is started simultaneous with or just after the beginning of the fill acquisition, depending on the site of injection (close to or further away from the relevant vasculature). Contrast is usually injected for 8 seconds so that each vascular segment in the arterial phase and early venous phase is filled for about $200^{\circ}$. If the rate (milliliters/second) of a contrast medium injection is too high, there will be downstream reflux of the contrast bolus. It has been shown that this phenomenon may prevent mixing of the bolus with the nonopacified blood arriving with each systole, thereby obscuring the pulsatile waveform normally seen in the time concentration curves of the $4 \mathrm{D}$ projections. Without the pulsatile information from the contrast bolus mixing with nonopacified blood between systole and diastole, it would be impossible to quantify blood flow using 4D-DSA data. ${ }^{11,12}$ Ruedinger et $\mathrm{al}^{12}$ reported that an injection protocol of $75 \%$ contrast (iopamidol, Isovue 370; Bracco) injected at $2.5 \mathrm{~mL} / \mathrm{s}$ for 8 seconds satisfies these requirements by producing strong pulsatility in the area of interest.

After a 4D acquisition (both mask and fill acquisitions) and reconstruction, a user has a 4 D-DSA vascular volume of $512 \times$ $512 \times 396$ voxels with resolution of $0.5 \mathrm{~mm}$. It may be viewed on the software as either a movie or interactively using a slider bar to progress through the sequences of timeframes (X Workplace; Siemens). Cropping, thresholding, and dual-volume visualizations of implanted devices are available to the user as well.

\section{D-DSA Reconstruction Workflow}

The reconstruction of $4 \mathrm{D}$-DSA uses the data from a conventional 3D-DSA rotational acquisition (fill run subtracted from the mask run) to create the vasculature geometry. This is then merged with the temporal information from the 2D-DSA projection images generated in the same acquisition. Figure 1 illustrates an overview of the automated reconstruction workflow. First, a thresholded 3D-DSA reconstruction, called a constraint volume, is generated (geometry). Next, for each acquisition angle (and time point), the measured 2D-DSA image is divided by a digital forward projection of the constraint volume. The resulting ratio images describe temporal changes in $\mathrm{x}$-ray intensity, relative to the constraint volume, due to the passage of contrast medium (temporal information). Finally, the temporal information, encoded in the sequence of ratio images, is individually back-projected onto the $3 \mathrm{D}$ constraint volume, producing a temporal sequence of $3 \mathrm{D}$ images. Each foreground voxel within the constraint volume is corrected by a function based on the ray-dependent ratio.

The result is a fully time-resolved 3D-DSA image (4D-DSA) of the anatomy of interest. The frame rate is identical to the acquisition frame rate (commonly 30 frames/second), and the total number of volumes is identical to the number of projection images in a rotation. For example, a 12 -second acquisition rotation would have 304 frames over $260^{\circ}$, yielding $>1$ frame per degree of rotation. Ultimately, a $4 \mathrm{D}$-DSA vascular volume allows viewing of the contrast bolus passage at any time and any desired viewing angle. 


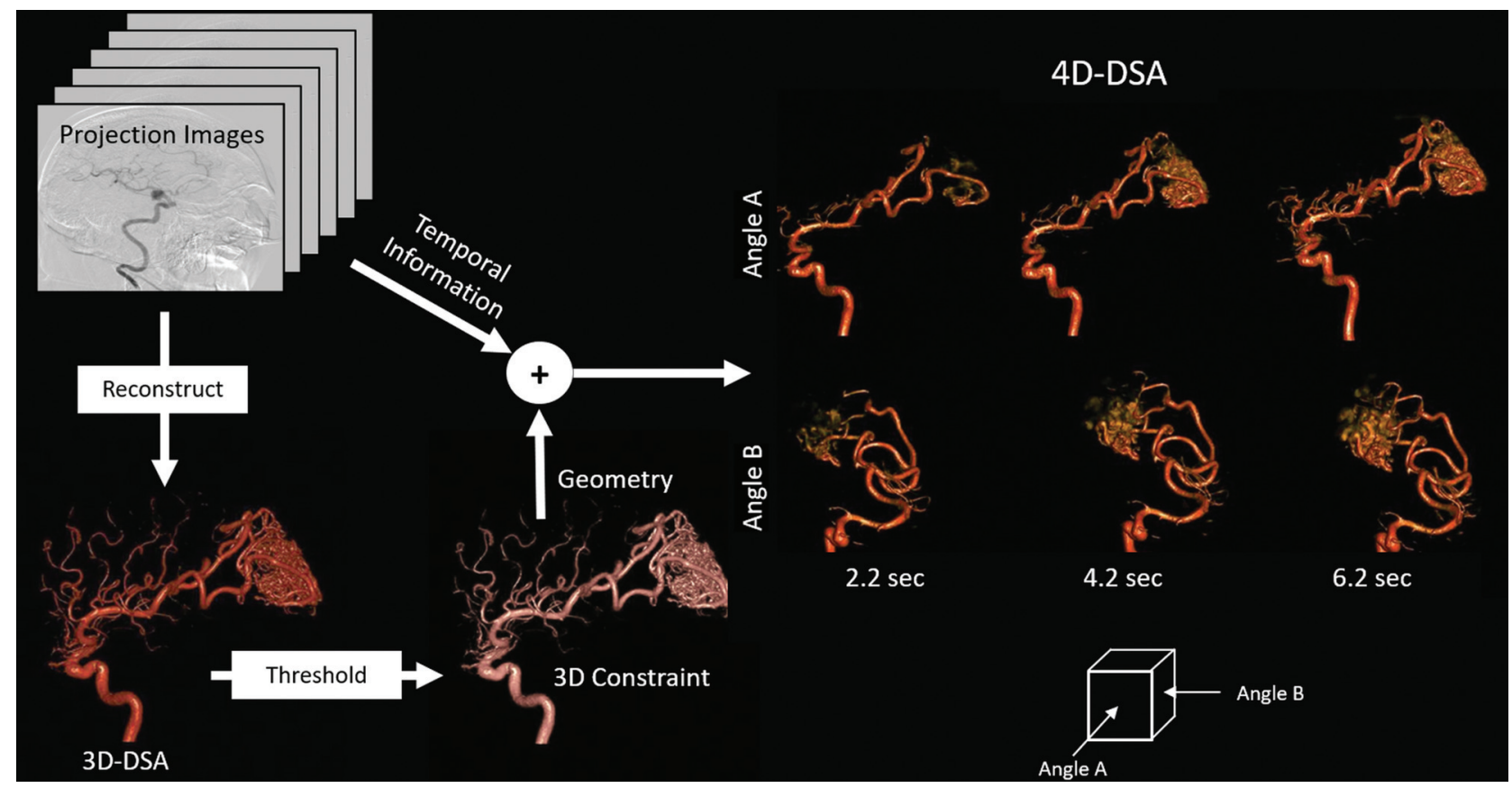

FIG 1. 4D-DSA reconstruction workflow. Starting at the upper left, projection images from a rotational acquisition are reconstructed into a 3DDSA. Following a threshold approach, a constraint volume is generated, which provides the geometric information for the 4D-DSA. Combining the constraint volume with the angle-specific temporal information results in the volumetric, time-resolved 4D-DSA volume.

\section{Clinical Applications of 4D-DSA}

In many instances, noninvasive imaging, ie, CTA or MRA, is sufficient to establish the presence and type of cerebrovascular anomalies; however, the superior spatial resolution of 2D- and 3D-DSA enhances the user's ability to understand some of the important details of the vascular anatomy. After 4D-DSA became commercially available in 2015, it further improved this capability.

AVMs and AVFs. In a preclinical study, Sandovol-Garcia et $\mathrm{al}^{13}$ compared the utility and accuracy of 4D-DSA with that of 2D- and 3D-DSA in a canine model. Angiographic data of the 3 techniques, showing both the intracranial and extracranial vasculature, were scored by 3 experienced neuroradiologists for the technique's ability to do the following: 1) display a series of previously determined vascular segments, 2) determine the direction of filling of the segment (antegrade or retrograde), and 3) determine the technique they would choose for performing the previous 2 tasks. In $74 \%$ of the datasets, $4 \mathrm{D}$-DSA was judged to be superior to the other methods. It was also reported that the use of the $4 \mathrm{D}$-DSA reconstruction alone would have resulted in a very meaningful reduction in both contrast dose and radiation exposure. ${ }^{13}$

Later reports by Sandovol-Garcia et $\mathrm{al}^{14}$ and by Lescher et $\mathrm{al}^{15}$ demonstrated the value of 4D-DSA in the diagnostic evaluation of AVMs. In the 2017 study of Sandovol-Garcia et al, ${ }^{16}$ the angioarchitecture from 2D, 3D, and 4D-DSA acquisitions of 6 AVMs was compared by 4 experienced observers. By consensus, the 4D-DSA studies provided the best ability to display the presence of intranidal aneurysms, fistulas, venous obstructions, and the sequence of filling and draining, all of which are important for diagnosis. ${ }^{16}$ Figure 2 illustrates the difficulty of 3D-DSA in viewing the angioarchitecture of the nidus due to vessel overlap and the improvement when viewing the nidus with the combined spatial and temporal features of $4 \mathrm{D}-\mathrm{DSA}$. Lescher et $\mathrm{al}^{15}$ also evaluated the utility of $2 \mathrm{D}, 3 \mathrm{D}$, and $4 \mathrm{D}$ reconstructions for displaying these same features. In both AVMs $(n=19)$ and AVFs $(n=9)$, the 4D-DSA studies provided the best detail of the angioarchitecture at the fistulous point or the nidus. Figure 3 illustrates the improved viewing of an AVF with time-resolved 4D-DSA.

In a single-center study of $26 \mathrm{AVMs}$, Lang et $\mathrm{al}^{17}$ confirmed the ability of 4D-DSA to show details of anatomy, as well as $2 \mathrm{D}$ images; additionally, nidal features of the AVMs were most clearly seen in the $4 \mathrm{D}$ reconstructions. On the basis of their observations, they postulated that the availability of the $4 \mathrm{D}$ application would decrease the number of $2 \mathrm{D}$ studies needed, therefore decreasing the radiation dose.

In addition to diagnosis, 4D-DSA added value over conventional techniques when used to create a treatment plan for AVMs for gamma knife radiosurgery (stereotactic radiosurgery). Chen et $\mathrm{al}^{18}$ compared the original treatment plans based on MR imaging and 2D-DSA with ones based on 4D-DSA in 20 consecutive patients with AVMs $(n=12)$ or AVFs $(n=8)$ who were scheduled for gamma knife radiosurgery. The radiosurgery treatment plans were overlaid, and the registration errors were compared. The determined area for treatment created with 4D-DSA was generally smaller and more clearly demonstrated the AVM nidus (in instances when it was supplied by multiple arteries) in comparison with the areas from conventional treatment plans. This study suggests that the use of $4 \mathrm{D}$-DSA for AVM radiosurgery could reduce the target volumes of irradiation, which may result in a decrease in the adverse effects of vascular radiosurgery. ${ }^{18}$

Intracranial Aneurysms. Endovascular treatment is now an option for most intracranial aneurysms. For safe and effective treatment, it is essential that both the relationship of an aneurysm 


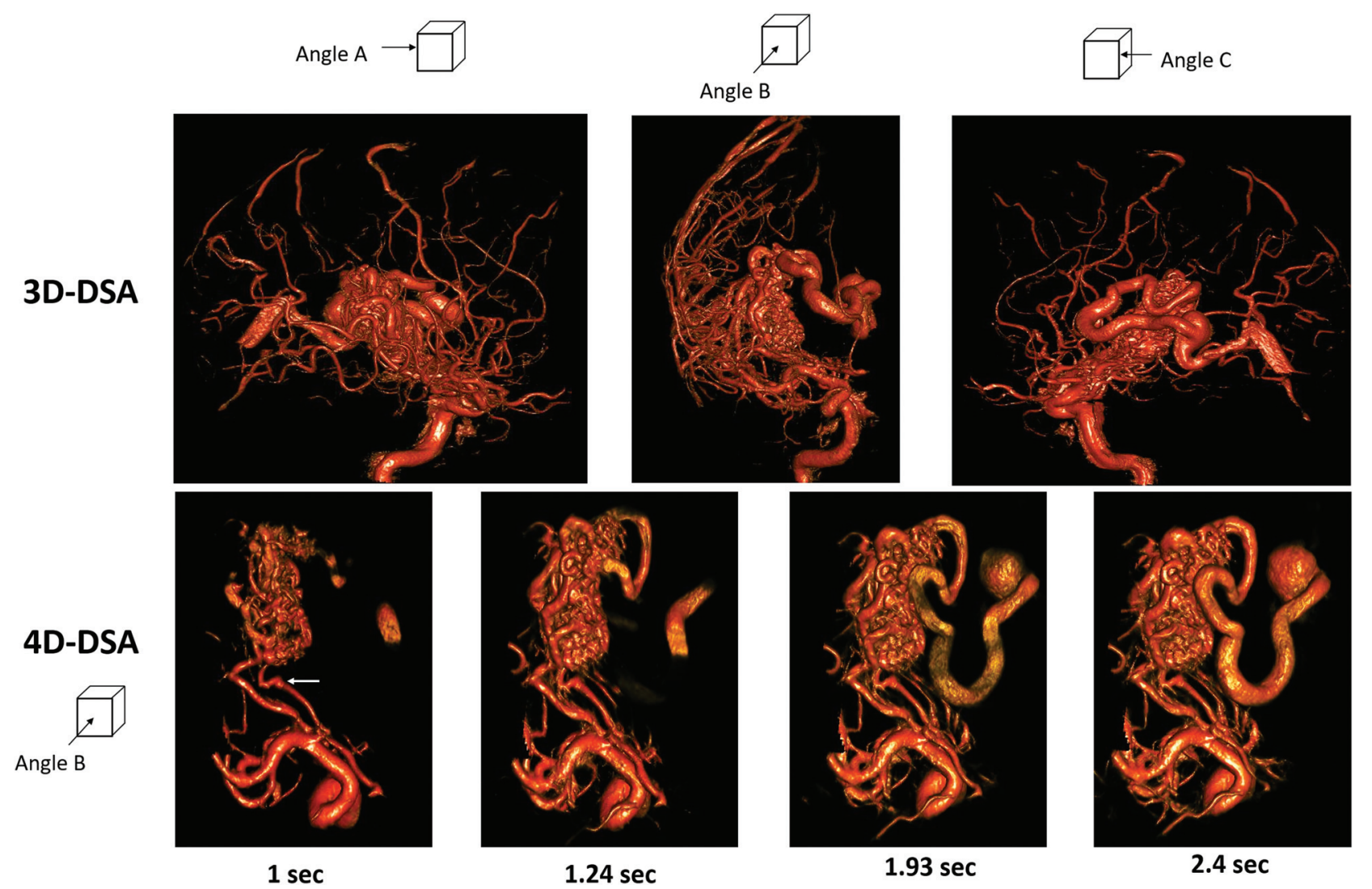

FIG 2. Three views from 3D-DSA of a patient with an AVM supplied by the lenticulostriate arteries (upper row). While the anatomic detail is excellent, vascular overlap in and around the nidus makes it impossible to see the angioarchitecture and to understand the sequences of blood flow into and out of the AVM. Early timeframes of the 4D-DSA (lower row) show details of the AVM nidus that are not visible on 3D-DSA. Note the small aneurysm on one of the lenticulostriate arteries (white arrow). No intranidal aneurysms are seen. Although the 4D-DSA images provided here are at 1 angle (angle B), these images may be viewed at any desired angle at any time of bolus passage.

t1 t2

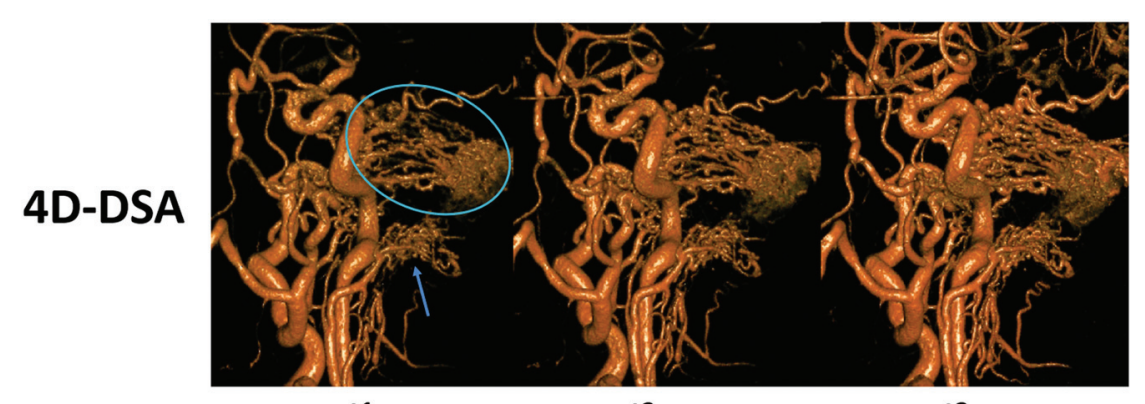

FIG 3. Three 4D-DSA images from early timeframes (tl-t3) of the filling of an AVF located along the intracranial surface of the petrous bone (blue circle). tl shows several small arteries supplying the AVF (blue arrow). In the image from $\mathrm{t} 3$, acquired less than a second later, these arteries are obscured. This information is helpful when trying to plan an endovascular approach to this abnormality.

As discussed earlier, the geometry obtained from 3D-DSA is used to create a 4D-DSA, so the geometric accuracy of the 3D-DSA reconstruction is essential for achieving accurate measurements from a 4D-DSA. Studies have shown the ability of 3D-DSA to accurately display the dimensions of intracranial vasculature, as well as intracranial aneurysm volume, with satisfactory precision. ${ }^{19,20}$ In an effort to further improve the precision of dimensional measurements obtained from 4D-DSA, Ruedinger et $\mathrm{al}^{21}$ evaluated the impact of various commercial reconstruction parameters on the accuracy of these measurements. Vessel reconstructions to the parent artery and to adjacent branches and the characteristics of the aneurysm be precisely defined. The accuracy of this information depends on the imaging technique chosen and the reconstruction tools used. Figure 4 illustrates a case with an intracranial aneurysm in which the spatial and temporal data obtained from 4D-DSA provide the ability to see the aneurysm in an unobstructed view, which is not possible with 3D-DSA modalities. were performed using edge enhancement or Hounsfield unit kernels and normal or smooth image characteristics. Aneurysm volume, surface area, dome height, and minimum and maximum ostium diameter measurement were made on 5 patient-specific 3D printed aneurysm models. The actual dimensions were determined from microCT measurements of the models, and it was concluded that reconstruction parameters had very little impact on aneurysm and vessel measurements. ${ }^{20} \mathrm{~A}$ subsequent article by this same group 
3D-DSA

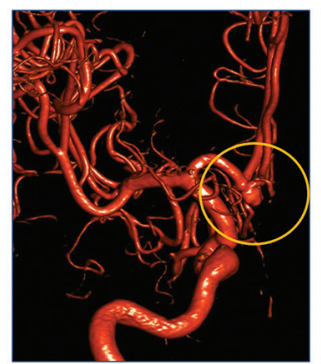

Anterior

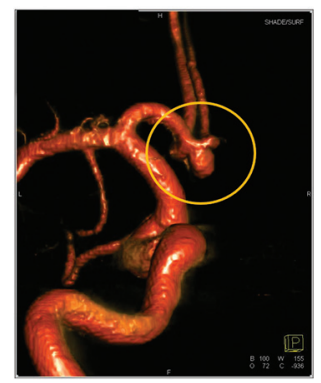

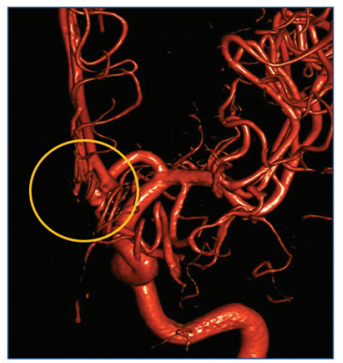

Posterior

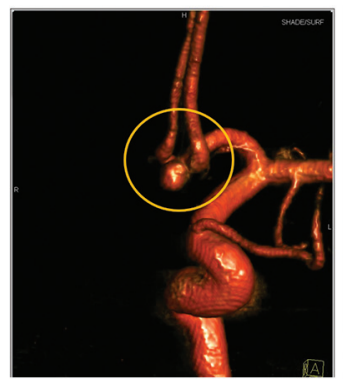

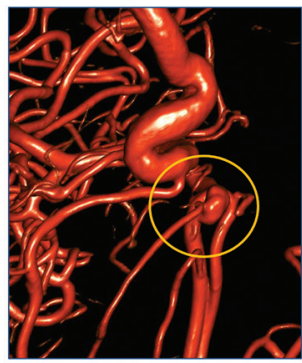

Cranial-Caudal View

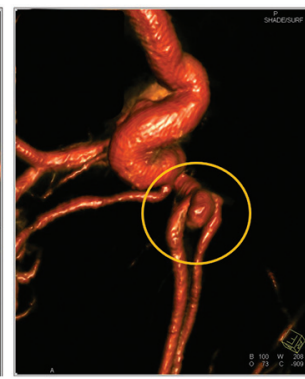

FIG 4. An anterior communicating artery aneurysm viewed from the anterior, posterior, and cranial-caudal positions of a 3D-DSA (upper row) and a 4D-DSA (lower row). The branches that obscure the view of the aneurysm (yellow circle) in the 3D-DSA are not yet filled in the 4D-DSA image, making the aneurysm neck and its relationship to adjacent branches visible. This information aids in the endovascular treatment of intracranial aneurysms. curves (TDCs) of 4D-DSA (also called time-concentration curves). Each voxel contains a TDC depicting bolus inflow, steady state, and bolus outflow. As the bolus moves through the vasculature, cardiac-derived oscillation in a ratio of contrast medium to nonopacified blood is observed. This oscillation superimposes on the TDCs as a quasi-sine wave (Fig 5). Investigating the TDCs shows a position-dependent temporal shift between the contrast-arrival time and the phase of the contained quasi-sine curve. Figure 5 illustrates a simplified schematic of how blood flow is quantified using 4D-DSA.

This effect, as first identified by Waechter et $\mathrm{al}^{24}$ can be used to derive velocity and flow of the liquid. Waechter et al used a $2 \mathrm{D}$ representation of the contrast agent over a given vessel segment and fit virtual maps representing a given velocity and flow to the measured data. For measuring the data, the vessel centerline and radii are first determined in 3D. Subsequently, a showed that when using a proper protocol for the injection of contrast medium, both the spatial information of 3D-DSA (geometry) and the temporal information of 4D-DSA (flow) remained highly accurate. ${ }^{12}$ Additionally, Sandoval-Garcia et $\mathrm{al}^{16}$ also found complete agreement in diagnostic characteristics in images from 2D-, 3D-, and 4D-DSA datasets. For endovascular treatment of aneurysms, it is critical that precise information on vessel sizes, ostium characteristics, and parent artery branch relationships be available.

Occlusive Disease. There are few reports about the use of $4 \mathrm{D}$ DSA in the diagnosis or treatment-planning of patients with occlusive disease. However, in patients for whom stent placement or angioplasty is indicated, the ability to make measurements from images with better spatial resolution than either CTA or MRA improves the ability to select optimal stent and balloon sizes. In a series of 24 patients with $(n=6)$ and without $(n=18)$ an MCA stenosis, Kammerer et $\mathrm{al}^{22}$ compared the utility of 4D-DSA with that of 3D-DSA for displaying the anatomy of the lenticulostriate arteries. Overall, the 4D-DSA reconstructions provided a superior display of the lenticulostriate artery anatomy; in patients with a chronic MCA stenosis, the 4D images also showed connections to the collateral network. The ability to view any time point in the contrast filling of a time-resolved 3D geometry was advantageous, compared with a conventional plain $3 \mathrm{D}$ geometry. ${ }^{22}$

\section{Quantification of Blood Flow from 4D-DSA}

The temporal information contained in 4D-DSA is sufficient to quantify blood velocity, and when combined with accurate vessel measurements, blood flow can be quantified. ${ }^{12,23}$ Flow derivation is based on identifying the time needed for a contrast bolus to pass between 2 points; this movement is captured in the time-density
2D flow map is determined showing the TDC of a given centerline voxel along the y-axis and displaying each centerline voxel in directional order. Then, by means of mathematic models of the blood flow, contrast injection, and fluid mixture and propagation, a simulated flow map is determined. The final step is error minimization between the simulated and measured flow map.

$\mathrm{Wu}$ et $\mathrm{al}^{25}$ applied a shifted least-squares analysis to time curves in a given phantom vessel segment. The vessel centerline and radii of each vessel segment of interest were determined at discrete intervals, and TDCs were recorded. Each TDC was shifted with respect to all other TDCs, and the temporal shift yielding the minimum least-squares difference was computed. A velocity for the segment was determined from temporal shift and spatial distance information with a relative root-mean-square error of $11 \%$ and a Pearson correlation coefficient with phase contrast (PC)-MR imaging of $r=0.835 .^{25}$

Shaughnessy et $\mathrm{al}^{26}$ derived a similar shift from the phase signal in the Fourier domain to determine liquid velocities in a given $3 \mathrm{D}$-printed, patient-specific vessel segment. In each vessel segment, the vessel centerline, discrete distances, and radii were determined. TDCs for each vessel centerline point were Fouriertransformed, and the fundamental frequency and associated phases were determined. The temporal shift of the TDC between 2 vessel centerline points was determined as the phase shift at the TDC fundamental frequency. This work yielded velocities within $10 \%$ of the ultrasonic flow meter and was extended to include a mass-preservation constraint to establish preserved flow across multiple bifurcations. ${ }^{26,27}$

In a study of AVMs, Lin et $\mathrm{al}^{28}$ showed the feasibility of measuring blood velocity in the angiography suite using $4 \mathrm{D}$-DSA. The study used a similar approach by taking the pulsatile information 

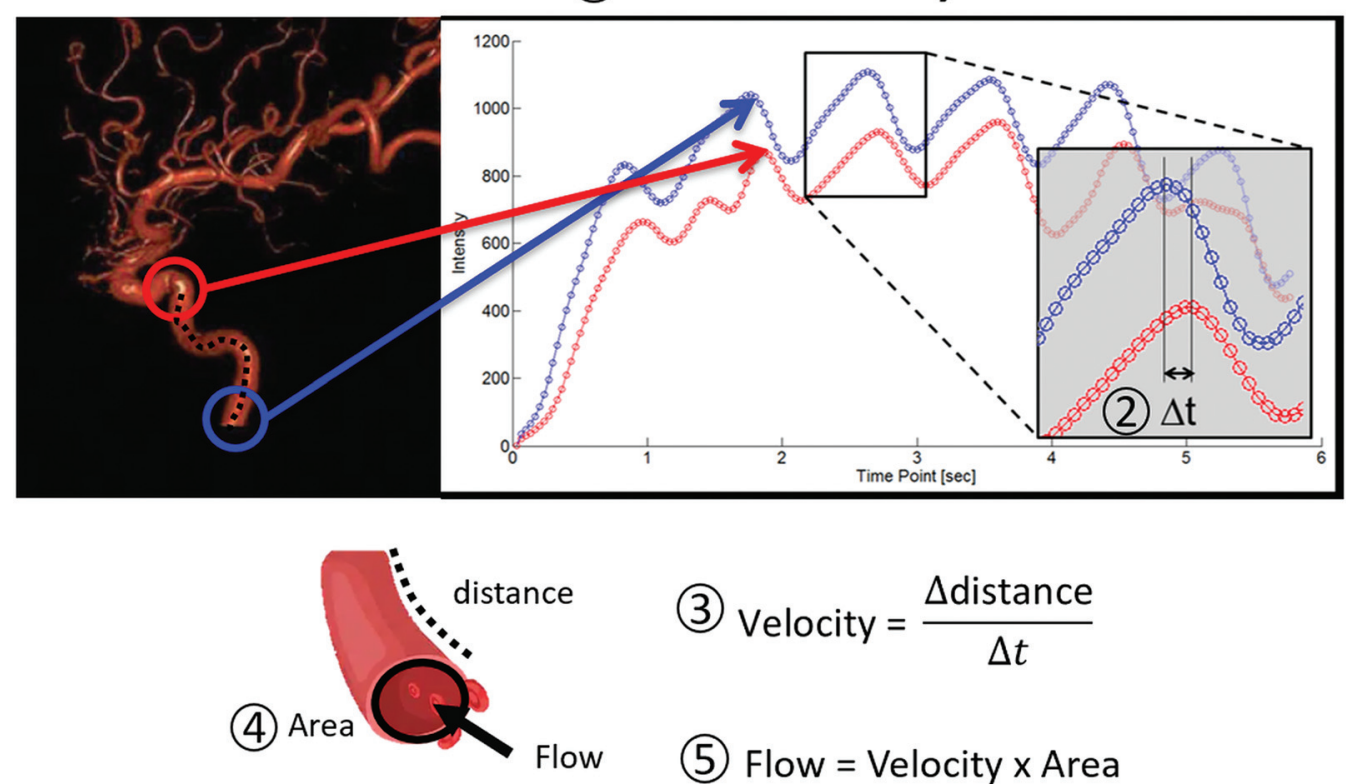

(3) Velocity $=\frac{\Delta \text { distance }}{\Delta t}$

(5) Flow = Velocity $\times$ Area

FIG 5. Simplified schematic of how flow is quantified using 4D-DSA: 1) Variations in the contrast density are due to the mixing of contrast with nonopacified blood at and downstream from the injection site with each cardiac cycle. These variations can be tracked in a time density curve (TDC) at any point along the vessel. 2) The time it takes the contrast bolus to arrive at a more distal location is quantified by the time-shift between the peak of the curves $(\Delta t)$. 3) Velocity is calculated by knowing the distance and time between 2 points in the vasculature. 4) The area of the vessel is calculated from the 4D-DSA geometry. 5) The flow is quantified using the velocity and area.

of the TDC and determining the peak-to-peak distance in ICA segments. With the temporal shift, as well as distance and vessel diameter determined, the contrast/blood mixture velocity and flow were derived. Evaluation of hemorrhagic-versus-nonhemorrhagic AVMs showed an increased velocity and flow in the hemorrhagic group, albeit the results were not statistically significant. To our knowledge, it is not known how vascular overlap may affect the accuracy of flow quantification. All methods mentioned above have been evaluated in noncommercial implementations of 4D-DSA algorithms and are currently not clinically available.

\section{Limitations and Helpful Solutions}

As is the case for 3D-DSA, patient motion, improper timing of the contrast injection, and involuntary vessel and muscle movement can all degrade the spatial and temporal information contained in a 4D-DSA reconstruction. If the injection sequence is used appropriately and no patient motion occurs, Lang et $\mathrm{al}^{29}$ showed that the geometry of the vasculature from a 4D-DSA is as reliable as conventional 3D-DSA. As briefly mentioned, vascular overlap may occur in $\geq 1$ of the $2 \mathrm{D}$ projections acquired during a rotational acquisition, which may have an impact on some of the temporal information in the $4 \mathrm{D}$ volume of the projection. Nonetheless, the added temporal dimension in a $4 \mathrm{D}$-DSA means that the clinician is no longer forced to view vasculature solely at the time of maximum contrast enhancement, as is the case in a conventional 3DDSA. By selecting different timeframes of bolus passage and viewing angles for image display, one can separate vascular structures that fill at different time points and that would have previously been obscured by overlap on many 2D projections and 3D images.

As previously mentioned, there is a distinct overlap phenomenon, arising at specific $2 \mathrm{D}$ projection angles during the acquisition when 2D images of superimposed contrast-enhanced arteries and veins are captured. Although these structures may be spatially separated in the 4D-DSA reconstruction and not visually overlapped, there is a possibility that, in some voxels, there may be mixing of temporal information at certain time points. The degree to which this affects flow quantification has not, to our knowledge, been defined. To resolve the ambiguity, Huizinga et $\mathrm{al}^{30}$ detailed, in a recent publication, the use of a novel reconstruction algorithm that uses a logical flow constraint. The algorithm enforces a connectivity between filling vasculature segments, removing erroneous early filling in distal vasculature by requiring a previously filled connected vessel.

From the perspective of a clinician using 4D-DSA for diagnosis and treatment-planning, postprocessing techniques have been found useful to further reduce the limitations caused by vascular overlap. One example is when vessels from one vascular territory are, in many viewing angles, superimposed on those from another territory, eg, MCA territory on ACA territory. We refer to this type of overlap as "superimposition," which is different from the vascular overlap that occurs when vessels in such close proximity can only be separated by viewing $4 \mathrm{D}$ timeframes acquired before the overlapping vessels are opacified. Superimposition can usually be resolved by simply cutting out the part of the $4 \mathrm{D}$ volume that is not of clinical interest, eg, the ACA territory when trying to view the details of an MCA aneurysm.

Another limitation of using a 4D-DSA volume occurs when a user thresholds an entire $4 \mathrm{D}$ volume. The optimal threshold for arteries will not be the same as those for veins because of the differences in contrast concentration and volume. Likewise, the optimal threshold for large arteries and veins will be different from that for 
small arteries or veins. By using the postprocessing tool to cut out a small ROI from the $4 \mathrm{D}$ volume and then thresholding it, one can view the anatomic and blood flow features; this outcome is not possible when thresholding the entire $4 \mathrm{D}$ volume at once.

\section{Summary}

4D-DSA, first developed in 2013, is a commercially available angiographic technique that provides the user with a fully time-resolved $3 \mathrm{D}$ volume of the vasculature. The ability to see any desired viewing angle at any desired time of bolus passage adds benefit over conventional 2D- and 3D-DSAs for cerebrovascular diseases.

Disclosures: Sebastian Schafer-UNRELATED: Employment: Siemens Healthineers, Comments: I am a full-time employee. Michael A. Speidel-RELATED: Grant: National Institutes of Health, Siemens Healthineers, Comments: Partial financial support was received from National Institutes of Health grant No R01HL116567 and a sponsored research agreement with Siemens Healthineers*; UNRELATED: Grants/ Grants Pending: Principal Investigator on National Institutes of Health grant No. R21EB023008 and a sponsored research agreement with Siemens Healthineers (both outside the scope of the present work)*; Patents (Planned, Pending or Issued): coinventor on US Patent 10,002,445 B2 related to 4D-DSA, and coinventor on 3 patents/patents pending that are outside the scope of the present work.* *Money paid to the institution.

\section{REFERENCES}

1. Vertinsky AT, Schwartz NE, Fischbein NJ, et al. Comparison of multidetector CT angiography and MR imaging of cervical artery dissection. AJNR Am J Neuroradiol 2008;29:1753-60 CrossRef Medline

2. Veldhoen S, Schöllchen M, Hanken H, et al. Performance of conebeam computed tomography and multidetector computed tomography in diagnostic imaging of the midface: a comparative study on phantom and cadaver head scans. Eur Radiol 2017;27:790-800 CrossRef Medline

3. Fahrig R, Fox AJ, Lownie S, et al. Use of a C-arm system to generate true three-dimensional computed rotational angiograms: preliminary in vitro and in vivo results. AJNR Am J Neuroradiol 1997;18:1507-14 Medline

4. Fahrig R, Holdsworth DW. Three-dimensional computed tomographic reconstruction using a C-arm mounted XRII: image-based correction of gantry motion nonidealities. Med Phys 2000;27:30-38 CrossRef Medline

5. Davis $B$, Royalty $K$, Kowarschik $M$, et al. 4D digital subtraction angiography: implementation and demonstration of feasibility. AJNR Am J Neuroradiol 2013;34:1914-21 CrossRef Medline

6. Akpek S, Brunner T, Benndorf G, et al. Three-dimensional imaging and cone beam volume $\mathrm{CT}$ in $\mathrm{C}$-arm angiography with flat panel detector. Diagn Interv Radiol 2005;11:10-13 Medline

7. Baba R, Konno Y, Ueda K, et al. Comparison of flat-panel detector and image-intensifier detector for cone-beam CT. Comput Med Imaging Graph 2002;26:153-18 CrossRef Medline

8. Schmitt H, Grass M, Suurmond R, et al. Reconstruction of blood propagation in three-dimensional rotational $x$-ray angiography (3D-RA). Comput Med imaging Graph 2005;29:507-20 CrossRef Medline

9. Copeland AD, Mangoubi RS, Desai MN, et al. Spatio-temporal data fusion for $3 \mathrm{D}+\mathrm{T}$ image reconstruction in cerebral angiography. IEEE Trans Med Imaging 2010;29:1238-51 CrossRef Medline

10. Mistretta CA. Sub-Nyquist acquisition and constrained reconstruction in time resolved angiography. Med Phys 2011;38:297585 CrossRef Medline

11. Lieber BB, Sadasivan C, Hao Q, et al. The mixability of angiographic contrast with arterial blood. Med Phys 2009;36:5064-78 CrossRef Medline

12. Ruedinger KL, Harvey EC, Schafer S, et al. Optimizing the quality of 4D-DSA temporal information. AJNR Am J Neuroradiol 2019;40:212429 CrossRef Medline
13. Sandoval-Garcia C, Royalty K, Aagaard-Kienitz B, et al. A comparison of 4D DSA with 2D and 3D DSA in the analysis of normal vascular structures in a canine model. AJNR Am J Neuroradiol 2015;36:195963 CrossRef Medline

14. Sandoval-Garcia C, Royalty K, Yang P, et al. 4D DSA a new technique for arteriovenous malformation evaluation: a feasibility study. J Neurointerv Surg 2016;8:300-04 CrossRef Medline

15. Lescher S, Gehrisch S, Klein S, et al. Time-resolved 3D rotational angiography: display of detailed neurovascular anatomy in patients with intracranial vascular malformations. J Neurointerv Surg 2017;9:88794 CrossRef Medline

16. Sandoval-Garcia C, Yang P, Schubert T, et al. Comparison of the diagnostic utility of 4D-DSA with conventional 2D- and 3D-DSA in the diagnosis of cerebrovascular abnormalities. AJNR Am J Neuroradiol 2017;38:729-34 CrossRef Medline

17. Lang S, Gölitz P, Struffert T, et al. 4D DSA for dynamic visualization of cerebral vasculature: a single-center experience in 26 cases. AJNR Am J Neuroradiol 2017;38:1169-76 CrossRef Medline

18. Chen KK, Guo WY, Yang HC, et al. Application of time-resolved 3D digital subtraction angiography to plan cerebral arteriovenous malformation radiosurgery. AJNR Am J Neuroradiol 2017;38:74046 CrossRef Medline

19. Yang P, Schafer S, Royalty K, et al. Measurement in the angiography suite: evaluation of vessel sizing techniques. J Neurointerv Surg 2016;8:965-68 CrossRef Medline

20. Bescós JO, Slob MJ, Slump CH, et al. Volume measurement of intracranial aneurysms from 3D rotational angiography: improvement of accuracy by gradient edge detection. AJNR Am J Neuroradiol 2005;26:2569-72 Medline

21. Ruedinger KL, Rutkowski DR, Schafer S, et al. Impact of image reconstruction parameters when using $3 \mathrm{D}$ DSA reconstructions to measure intracranial aneurysms. J Neurointerv Surg 2018;10:285-89 CrossRef Medline

22. Kammerer S, Mueller-Eschner M, Berkefeld J, et al. Time-resolved 3D rotational angiography (4D DSA) of the lenticulostriate arteries: display of normal anatomic variants and collaterals in cases with chronic obstruction of the MCA. Clin Neuroradiol 2017;27:451-57 CrossRef Medline

23. Xu J, Shaughnessy G, Schafer S, et al. Assessing the reliability of pulsatility in four-dimensional digital subtraction angiography time concentration curves. Biomed Phys Eng Express 2018;4:35021 CrossRef

24. Waechter I, Bredno J, Hermans R, et al. Model-based blood flow quantification from rotational angiography. Med Image Anal 2008;12:586602 CrossRef Medline

25. Wu Y, Shaughnessy G, Hoffman CA, et al. Quantification of blood velocity with $4 \mathrm{D}$ digital subtraction angiography using the shifted leastsquares method. AJNR Am J Neuroradiol 2018;39:1871-77 CrossRef Medline

26. Shaughnessy G, Schafer S, Speidel MA, et al. Measuring blood velocity using 4D-DSA: a feasibility study. Med Phys 2018;45:4510-18 CrossRef Medline

27. Shaughnessy G, Hoffman C, Schafer S, et al. Precision blood flow measurements in vascular networks with conservation constraints. In: Medical Imaging 2018: Image-Guided Procedures, Robotic Interventions, and Modeling. Vol 10576. International Society for Optics and Photonics; 2018;105762Q CrossRef

28. Lin CJ, Yang HC, Chien AC, et al. In-room assessment of intravascular velocity from time-resolved rotational angiography in patients with arteriovenous malformation: a pilot study. J Neurointerv Surg 2018;10:583-88 CrossRef Medline

29. Lang S, Hoelter P, Birkhold AI, et al. Quantitative and qualitative comparison of 4D-DSA with 3D-DSA using computational fluid dynamics simulations in cerebral aneurysms. AJNR Am J Neuroradiol 2019;40:1505-10 CrossRef Medline

30. Huizinga N, Keil F, Birkhold A, et al. 4D flat panel conebeam CTA for in vivo imaging of the microvasculature of the human cortex with a novel software prototype. AJNR Am J Neuroradiol 2020;41:976-79 CrossRef Medline 